\title{
A Study on Possibility to Apply Piezoelectric Actuators for Active Reduction of Torsional Beams Vibrations
}

\author{
E. Augustyn And M.S. Kozien** \\ Cracow University of Technology, Institute of Applied Mechanics \\ al. Jana Pawła II 37, 31-864 Kraków, Poland
}

\begin{abstract}
In this paper, the results of several tests concerning possible application of piezoelectric elements to reduce torsional vibrations of a beam are presented and discussed. The proposed method of application is a novel one. The piezoelectric elements are positioned in two pairs and glued to the beam at the chosen cross-section. These elements are activated using a harmonically varying voltage of the same amplitude and opposite in phase. Simulations are performed regarding the active reduction of the lowest natural frequencies of vibration of the fixed-free beam with the use of piezoelectric actuators. The results of these simulations are obtained by means of the finite element method (ANSYS).
\end{abstract}

DOI: 10.12693/APhysPolA.125.A-164

PACS: 33.15.Mt, 34.50.Ez, 77.22.Gm

\section{Introduction}

Some structural engineering elements present in a natural way a one dimensional, beam-like type of behavior. For example, turbine blades belong to such a kind of elements in machine dynamics. Due to external excitations, these elements may start to vibrate [1-3]. These vibrations can be reduced in different ways $[1,2,4]$. In recent years, piezoelectric elements are applied to reduce vibrations, especially the bending vibrations of beams [5-7], in both active and passive systems [8-12].

Let us assume that the analyzed beam is prismatic. The form of the equations of motion of the vibrating beam, strongly depends on the shape of the cross-section. The most difficult in terms of analysis is the shape referred to as the "thin-walled type" (in closed or open form) [13-15]. The next group, much easier to analyze, is the monolithic cross-section type. The easiest for analysis, especially for the twisting case, are beams of circular type. Having in mind the possibility to reduce vibrations of turbine blades by means of piezoelectric actuators, the case of a solid cross-section of a general form is considered hereafter.

When the external exciting force has the form of a twisting moment (distributed or concentrated) or a force (distributed or concentrated), line of action of which crosses the shear center of the cross-section, the equations of motion of a beam can be separated. It means that twisting vibrations of the beam can be analyzed separately from the bending ones.

In the paper, the feasibility study of possible application of piezoelectric elements to reduce torsional vibration of the beam with monolithic cross section is performed. The aim of the paper is to verify an original idea of positioning piezoelectrics element as two pairs

*corresponding author; e-mail: kozien@mech.pk.edu.pl glued to the beam at a chosen cross-section and activating them by the harmonically variable voltage with the same amplitude but opposite in phase. Simulations are done using the finite element method (ANSYS) for computation of a fixed-free beam with rectangular crosssection in its lowest natural torsional mode. The novelty of the approach consists in analysis of the possibility of application of piezoelectric elements to reduce twisting vibrations of such a beam.

\section{Equation of motion}

A prismatic beam with monolithic cross-section is considered for analysis. Moreover, it is assumed that its material is isotropic. These assumptions make the analysis easier and do not restrict the possibility to reduce twisting vibrations of a beam by piezoelectric elements. The geometry of the cross-section is shown in Fig. 1, where $G$ is the gravity center and $S$ is the shear center. The origin of coorinate system $S x y z$ lies in shear center, and the axes $S y$ and $S z$ are parallel to the principal axes of the cross-section $(G \eta, G \zeta)$.

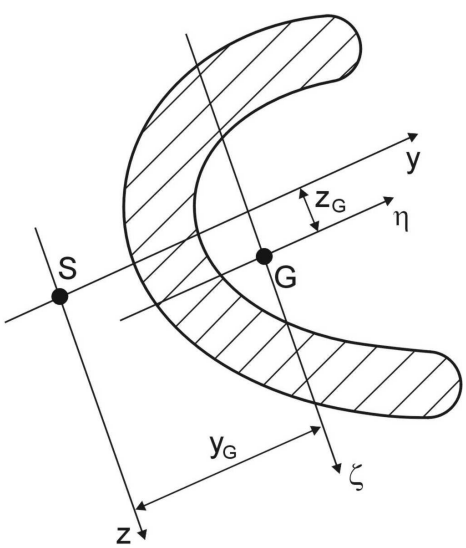

Fig. 1. Geometry of the cross-section. 
Equations of coupled vibrations of the beam takes the following form:

$$
\left\{\begin{array}{l}
E J_{\zeta} \frac{\partial^{4} v(x, t)}{\partial x^{4}}+\rho A\left[\frac{\partial^{2} v(x, t)}{\partial t^{2}}-z_{G} \frac{\partial^{2} \varphi(x, t)}{\partial t^{2}}\right]=q_{y}(x, t), \\
E J_{\eta} \frac{\partial^{4} w(x, t)}{\partial x^{4}}+\rho A\left[\frac{\partial^{2} w(x, t)}{\partial t^{2}}+y_{G} \frac{\partial^{2} \varphi(x, t)}{\partial t^{2}}\right]=q_{z}(x, t), \\
-G J_{s} \frac{\partial^{2} \varphi(x, t)}{\partial x^{2}}+\rho J_{0} \frac{\partial^{2} u(x, t)}{\partial t^{2}} \\
\quad+\rho A\left[y_{G} \frac{\partial^{2} w(x, t)}{\partial t^{2}}-z_{G} \frac{\partial^{2} v(x, t)}{\partial t^{2}}\right]=m_{s}(x, t),
\end{array}\right.
$$

where $v(x, t)$ is the displacement of the beam in $y$ direction, $w(x, t)$ is the displacement of the beam in $z$ direction, $\varphi(x, t)$ is the rotation of the axis of beam around $x$ direction, $J_{\eta}$ is the principal moment of inertia of the cross-section with respect to $\eta$ axis, $J_{\zeta}$ is the principal moment of inertia of the cross-section with respect to $\zeta$ axis, $J_{0}$ is the polar moment of inertia with respect to shear center, $J_{s}$ is the equivalent polar moment of inertia of the cross-section due to torsion, $E$ is the Young's modulus, $G$ is the shear modulus, $\rho$ is the material density, $y_{G}, z_{G}$ are coordinates of the gravity center $G$ in the coordinate system $S x y z, q_{y}(x, t)$ is the distributed force acting in $y$ direction (external excitation), $q_{z}(x, t)$ is the distributed force acting in $z$ direction (external excitation), and $m_{s}(x, t)$ is the distributed twisting moment (external excitation).

When the considered cross-section is such that coordinates of the shear center and those of the gravity center are the same (e.g. for the cross-section having two axes of symmetry), the relationship $y_{G}=z_{G}=0$ is valid. This means that the equation of motion for a twisting existing in Eq. (1) is separated from the equations of bending vibrations, thus taking a simplified form

$$
-G J_{s} \frac{\partial^{2} \varphi(x, t)}{\partial x^{2}}+\rho J_{0} \frac{\partial^{2} u(x, t)}{\partial t^{2}}=m_{s}(x, t) .
$$

This means that for the considered cross-section it is possible to analyze torsional vibrations independently from the bending ones.

\section{Action of pairs of piezoelectric elements on a beam in the static case}

All finite element method simulations were done for fixed-free beam with length $0.154 \mathrm{~m}$, width $0.022 \mathrm{~m}$ and height $0.0008 \mathrm{~m}$, made of steel (density $\rho=7800 \mathrm{~kg} / \mathrm{m}^{3}$, Young's modulus $E=210 \mathrm{GPa}$, Poisson ratio $\nu=0.29$, damping ratio 0.005$)$. The piezoelectric actuators had dimensions $0.01 \mathrm{~m}$ x $0.01 \mathrm{~m} \times 0.0006 \mathrm{~m}$ (see Fig. 2 for details). They were made of NEPEC, with piezoelectric properties described by:

$$
\underline{c}=\left[\begin{array}{cccccc}
13.2 & 7.1 & 7.3 & 0 & 0 & 0 \\
0 & 13.2 & 7.3 & 0 & 0 & 0 \\
0 & 0 & 11.5 & 0 & 0 & 0 \\
0 & 0 & 0 & 3.0 & 0 & 0 \\
0 & 0 & 0 & 0 & 2.6 & 0 \\
0 & 0 & 0 & 0 & 0 & 2.6
\end{array}\right] \cdot 10^{10}
$$

$$
\begin{aligned}
& \underline{e}=\left[\begin{array}{ccc}
0 & 0 & -4.1 \\
0 & 0 & -4.1 \\
0 & 0 & 14.1 \\
0 & 0 & 0 \\
0 & 10.5 & 0 \\
10.5 & 0 & 0
\end{array}\right] \\
& \underline{\varepsilon}=\left[\begin{array}{ccc}
804.6 & 0 & 0 \\
0 & 804.6 & 0 \\
0 & 0 & 569.7
\end{array}\right] \cdot 8.854,
\end{aligned}
$$

where $\underline{c}$ is the elastic constant matrix, $\underline{e}$ is the piezoelectric constant matrix, and $\underline{\varepsilon}$ is the permittivity constant matrix.

In the well-known literature applications, the reduction of the bending vibrations of a beam is obtained by pairs of piezoelectric elements glued to external surfaces of a beam [5-7]. After applying in-phase voltage of suitable amplitude to their external surfaces, they generate the anti-bending moment acting along the beam.

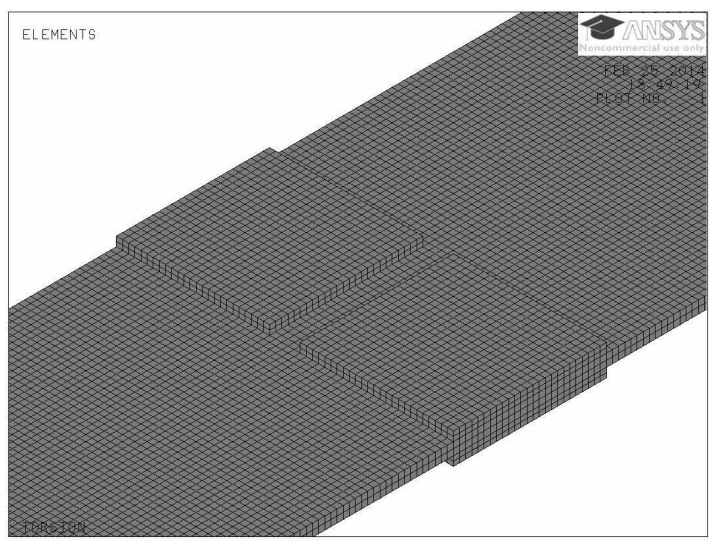

Fig. 2. Geometry of piezoelectric actuators in FEM model.

In the proposed application the piezoelectric elements are mounted (glued) to the beam in pairs along its axis to external surfaces of the beam in such way that they are distant by the same value from the fixed end. As a result, it is obtained the two separated twin-pairs of elements. For the tests, the centers of elements were distanced $0.079 \mathrm{~m}$ from the fixed end. Positions of the pairs are shown in FEM model in Fig. 2. The novelty of application consists in applying voltages opposite in phase to external surfaces of the piezoelectric elements in order to make them a bending moment across the line of beam. Results of application of piezoelements activated this way for the static case in the form of displacements of the beam are shown in Fig. 3 .

It should be noted that if the position of the one pair of elements is a little shifted with respect to the position of the second one, the bending case of vibrations will be observed due to the beam rigidity along beam being lower then this across the beam, however the voltages 


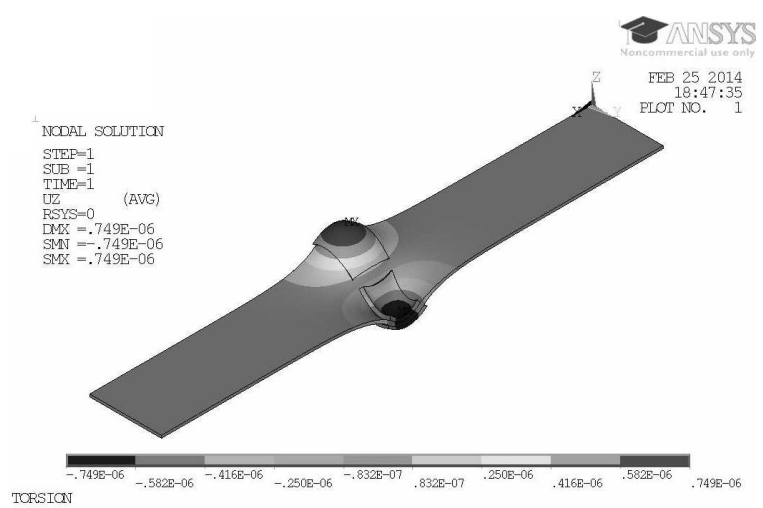

Fig. 3. Torsional type of deflection of the beam after action of two pairs of piezoelectric elements, equally distant from the fixed end and activated in opposite phases.

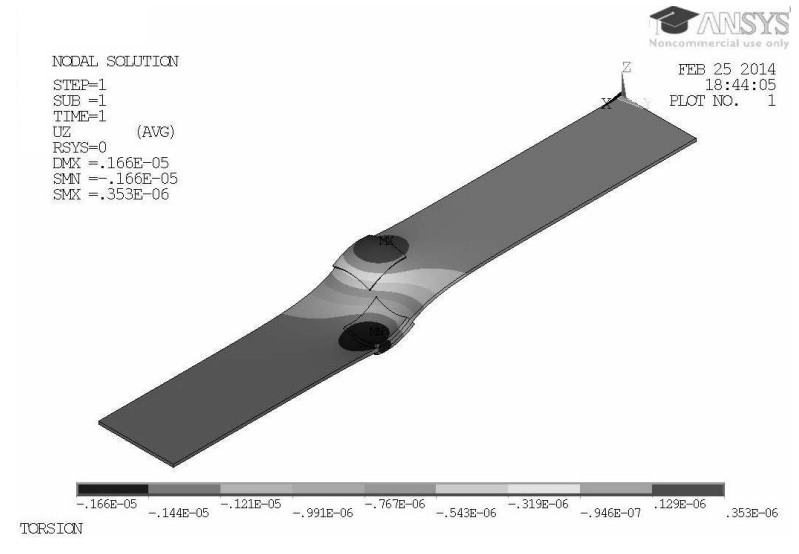

Fig. 4. Bending type of deflection of the beam after action of two pairs of piezoelectric elements, places at different distances from the fixed end and activated in opposite phases.

put to pairs of elements are in opposite phase. This kind of deformation is shown in Fig. 4. The centers of pairs of elements were distant by $0.079 \mathrm{~m}$ and $0.089 \mathrm{~m}$ from the fixed end, respectively.

If activation of piezoelectric elements by applied voltage as function of time depends on the beam vibration, they may produce an anti-twisting moment action of which consists in reducing torsional vibrations of the beam. Position of the pair of elements along the beam depends on the reduced form of vibration. In the next section, the results of action of elements distance of which from the fixed end of the beam is varying is discussed.

\section{FEM simulation}

At the beginning, the modal analysis allowed to identify eigenmodes and their natural frequencies was performed for the beam without piezoelectric elements. The obtained values of eigenfrequencies with description of the type of mode is given in Table. In Fig. 5, the form of the first torsional eigenmode is shown.

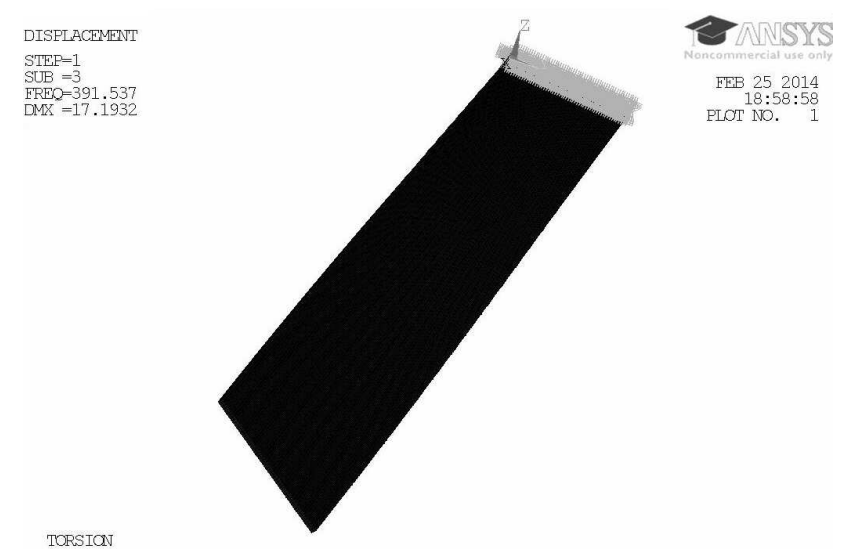

Fig. 5. The first torsional eigenmode $(f=391.5 \mathrm{~Hz})$.

TABLE

Types of natural modes and their frequencies.

\begin{tabular}{c|c|cc}
\hline \hline mode & frequency $[\mathrm{Hz}]$ & \multicolumn{2}{|c}{ type } \\
\hline 1 & 28.5 & bending & $w$ \\
2 & 178.6 & bending & $w$ \\
3 & 391.5 & torsional & $\varphi$ \\
4 & 500.7 & bending & $w$ \\
5 & 766.8 & bending & $v$ \\
6 & 983.2 & bending & $w$ \\
7 & 1186.9 & torsional & $\varphi$ \\
8 & 1629.1 & bending & $w$ \\
9 & 2018.4 & torsional & $\varphi$
\end{tabular}

To simulate reduction of torsional vibrations by piezoelectric elements, the steady-state case of vibrations was considered, separately for each mode. Vibrations were excited by the concentrated harmonic force acting perpendiculary to the surface of the beam in one its free corners. The force amplitude was $0.1 \mathrm{~N}$. Positions of the two pairs of piezoelectric elements were varying along the beam. Three values of the applied voltages were considred: $80 \mathrm{~V}, 100 \mathrm{~V}$ and $120 \mathrm{~V}$. The applied voltage signals were in phase with the acting force during simulation.

Four control points were chosen to measure the amplitude of vibration of the beam:

- (END) - the point near the place where the force was applied $(x=0.154 \mathrm{~m}, y=0.0011 \mathrm{~m}, z=0 \mathrm{~m})$;

- (MID) - the point in analogous position like point (END) in the middle of the beam $(x=0.077 \mathrm{~m}$, $y=0.0011 \mathrm{~m}, z=0 \mathrm{~m})$;

- (END-L) - the point on the line of the beam in the same cross-section as point (END) $-(x=0.154 \mathrm{~m}$, $y=0 \mathrm{~m}, z=0 \mathrm{~m})$;

- (MID-L) - the point lying on the line of a beam in the same cross-section as point (MID) $(x=$ $0.077 \mathrm{~m}, y=0 \mathrm{~m}, z=0 \mathrm{~m})$. 
Influence of the applied piezoelectric elements on reduction of torional vibrations of the beam were estimated by means of the relative amplitude reduction ratio. The relative reduction ratio is the ratio of the difference between amplitude of vibrations (transversal displacement) in a control point for the beam without piezoelectric elements and with elements to the value for the beam without piezoelectric elements. This value is given in percent.

The values of the obtained relative reduction ratios for different positions of piezoelectric elements along the beam are showm in Fig. 6 (for voltage amplitude $80 \mathrm{~V}$ ), in Fig. 7 (for voltage amplitude $100 \mathrm{~V}$ ) and in Fig. 8 (for voltage amplitude $120 \mathrm{~V}$ ). In the figures, position of elements is expressed as the distance of the nearest edges of pairs of elements from the fixed edge of beam.

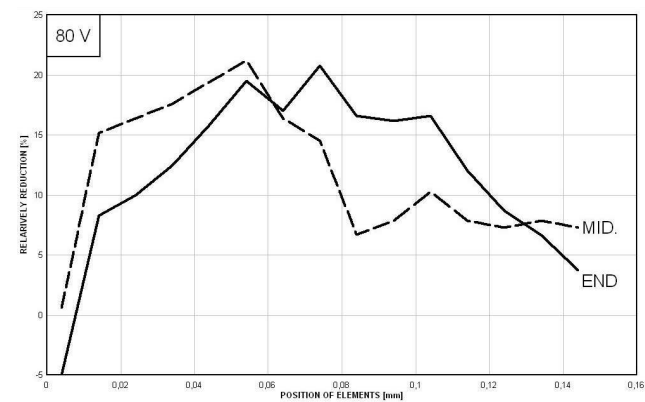

Fig. 6. The relative vibration reduction ratio value for voltage amplitude $80 \mathrm{~V}$.

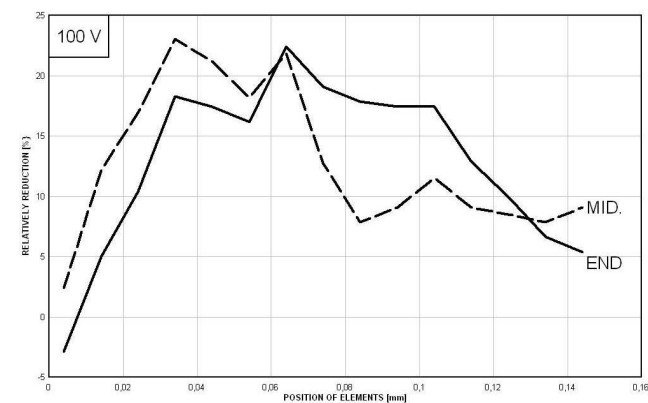

Fig. 7. The relative vibration reduction ratio value for voltage amplitude $100 \mathrm{~V}$.

When the piezoelectric elements were activated, transversal displacements od the beam in control points (END-L) and (MID-L) were close to zero. This means that during operation of piezoelectric elements, the bending vibrations were not excited.

The general conclusion that can be drawn from the presented results for the reduction ratio is that it is possible to reduce torsional vibrations, especially of thin-type beams, by application of piezoelectric elements which generate the torsional moment depending on its deformation. Different maximum values of the relative reduction ratio were obtained in the chosen control points for the same voltage amplitudes. Therefore, for actual applica-

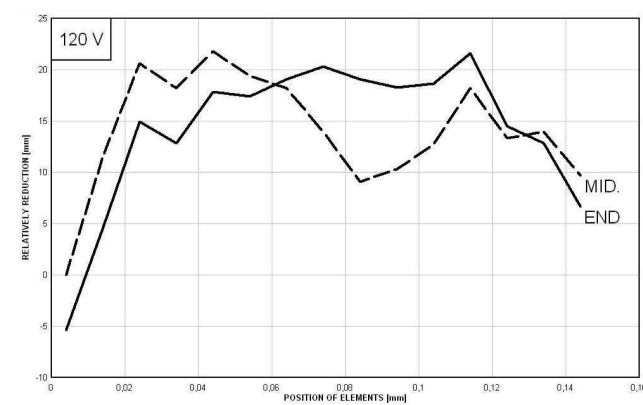

Fig. 8. The relative vibration reduction ratio value for voltage amplitude $120 \mathrm{~V}$.

tion it is important to define the criterion for vibration reduction. In case of practical applications, the problem of optimal position of elements and applied voltage are the main problems and must be considered. For the single-mode case, the general remark is that they should be positioned at the cross-section of the maximum twisting moment, as it is for the bending case $[8,9]$.

\section{Final remarks}

The results of feasibility studies of possibility to apply piezoelectric elements to reduce torsional vibrations of beams make possible to formulate the following remarks and conclusions:

- The proposed method of reduction of vibrations is original due to applied configuration of the piezoelectric elements.

- In general it is possible to reduce torsional vibration, specifically in thin beams type, by application of piezoelectric elements which generate the torsional moment due to their deformation.

- Due to possibility of changing of the voltage phase applied to piezoelectric elements in twin pairs, it is possible to apply them to reduce either torsionaltype vibrations or the bending ones.

- When the values of natural frequencies of the torsional mode and the bending mode are sufficiently different from each other, transverse vibrations of the beam are not excited by piezoelectric elements acting to reduce its torsional vibrations.

- In another realisation, one element of the pair may be an actuator and the second one may work as a sensor. It lowers the effectiveness of reduction.

- The problem of choosing the voltage amplitude (control algorihtm) and phase shift, for different types of beam response (steady-state/transient) depends on the type of analysis. Moreover, position of the elements along beam is still open problem for multimodal respone. 


\section{References}

[1] R. Gryboś, Vibrations of machines, Politechnika Śląska, Gliwice 1996, (in Polish).

[2] R. Łączkowski, Vibrations of elements of turbin blades, WNT, Warszawa 1974, (in Polish).

[3] J.S.Rao, Turbomachine Blade Vibration, Wiley, Chichester 1991.

[4] L. Pust, L. Pesek, J. Theor. Appl. Mech., submited, (2014).

[5] S.P. Elliott, P.A. Nelson, Active control of vibrations, Academic Press, London 1997.

[6] S.O.R. Moheimani, A.J. Fleming, Piezoelectric transducers for vibration control and damping, Springer, London 2006.

[7] A. Preumont, Mechatronics. Dynamics of Electromechanical and Piezoelectric Systems, Springer, Berlin 2006 .

[8] A. Brański, M. Borkowski, S. Szela Acta Phys. Pol. A 118, 17 (2010).
[9] A. Brański, G. Lipiński Acta Phys. Pol. A 119, 936 (2011).

[10] R. Filipek, J. Wiciak, Europ. Phys. J., ST 154, 57 (2008).

[11] M. Kozień, B. Koltowski, Acta Phys. Pol. A 119, 1005 (2011).

[12] M. Kozień, J. Wiciak, Acta Phys. Pol. A 116, 348 (2009).

[13] J.M. Gere J. Appl. Mech. Trans. ASME 21(4), 337 (1954).

[14] S. Piechnik, Technical mechanics of solids, Politechnika Krakowska, Cracow 2006.

[15] W.Z. Vlasow, Thin-walled elastic beams, Gos. Izd. Fiz. Mat. Literat., Moskva (1959), (in Russian). 